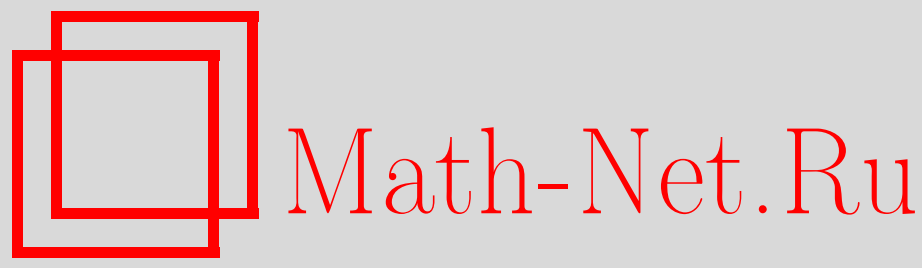

О. К. Пашаев, Релятивистские нелинейные уравнения Шредингера и Бюргерса, ТМФ, 2009, том 160, номер 1, 178-188

DOI: https://doi.org/10.4213/tmf6389

Использование Общероссийского математического портала Math-Net.Ru подразумевает, что вы прочитали и согласны с пользовательским соглашением http://www . mathnet.ru/rus/agreement

Параметры загрузки:

IP : 54.174 .149 .18

26 апреля 2023 г., $07: 44: 02$

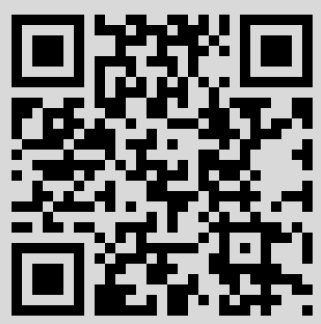




\title{
ФИЗИКА
}

Том 160, № 1

июль, 2009

2009 г.

\author{
O. К. Пашаев*
}

\section{РЕЛЯТИВИСТСКИЕ НЕЛИНЕЙНЫЕ УРАВНЕНИЯ ШРЕДИНГЕРА И БЮРГЕРСА}

Построены релятивистские комплексные уравнение Бюргерса-Шредингера и нелинейное уравнение Шредингера. В нерелятивистском пределе они переходят соответственно в обычное уравнение Бюргерса и в обычное нелинейное уравнение Шредингера. Эти уравнения оказываются интегрируемыми во всех порядках разложения по релятивистским поправкам.

Ключевые слова: квазирелятивистское нелинейное уравнение Шредингера, релятивистское уравнение Бюргерса-Шредингера, иерархия нелинейного уравнения Шредингера, релятивистская дисперсия, $q$-производная, рекурсионный оператор.

\section{1. ОБЩАЯ ИЕРАРХИЯ БЮРГЕРСА-ШРЕДИНГЕРА}

Релятивистское линейное уравнение Шредингера обсуждалось на ранних этапах развития квантовой механики, но впоследствии было вытеснено уравнениями Клейна-Гордона и Дирака. Недавно релятивистские варианты уравнения Шредингера рассматривались при исследовании релятивистских связанных состояний типа кварк-антикварк [1] и гравитационного коллапса бозонной звезды [2]. Нелинейный вариант модели встречался в виде квазирелятивистского уравнения ХартриФока [3]. Тем не менее ни одна из этих моделей не оказалась интегрируемой. В настоящей работе строится интегрируемое релятивистское нелинейное уравнение Шредингера (НУШ), свойство интегрируемости которого сохраняется во всех порядках разложения по $1 / c$.

Рассмотрим уравнение Шредингера в размерности $1+1$ для свободной частицы с классической дисперсией общего аналитического вида $E=E(p)$

$$
i \hbar \frac{\partial \Psi}{\partial t}=\mathcal{H}\left(P_{1}\right) \Psi
$$

где $P_{0}=i \hbar \partial / \partial t$ и $P_{1}=-i \hbar \partial / \partial x$ представляют собой соответственно операторы сдвигов по времени и пространству, коммутирующие с оператором Шредингера $S=i \hbar \partial / \partial t-\mathcal{H}\left(P_{1}\right),\left[P_{\mu}, S\right]=0, \mu=0,1$. Оператор общего преобразования буста,

${ }^{*}$ Department of Mathematics, Izmir Institute of Technology, Urla-Izmir, Turkey. E-mail: oktaypashaev@iyte.edu.tr 
определяемый как $K=x-t \mathcal{H}^{\prime}\left(P_{1}\right)$, также коммутирует с $S,[K, S]=0$. Вычисляя его коммутаторы с операторами пространственных и временны́х сдвигов, получаем алгебру операторов симметрии

$$
\left[P_{0}, P_{1}\right]=0, \quad\left[P_{0}, K\right]=-\hbar \mathcal{H}^{\prime}\left(P_{1}\right), \quad\left[P_{1}, K\right]=-i \hbar .
$$

Тем самым, если $\Psi$ - решение уравнения (1) и $W$ - оператор из указанной алгебры, т.е. $[W, S]=0$, то $W \Psi$ также оказывается решением уравнения (1).

При заданной классической дисперсии $E=E(p), E_{0} \equiv E(0)$ определим $E$-многочлены $H_{n}^{(E)}(x, t)$ с помощью производящей функции

$$
\exp \left[\frac{i}{\hbar}\left(p x-\left(E(p)-E_{0}\right) t\right)\right]=\sum_{n=0}^{\infty}\left(\frac{i}{\hbar}\right)^{n} \frac{p^{n}}{n !} H_{n}^{(E)}(x, t),
$$

или, эквивалентно,

$$
H_{n}^{(E)}(x, t)=\exp \left[-\frac{i}{\hbar}\left(\mathcal{H}\left(-i \hbar \frac{\partial}{\partial x}\right)-E_{0}\right) t\right] x^{n} .
$$

Многочлен $H_{n}^{(E)}(x, t)$ оказывается решением уравнения

$$
i \hbar \frac{\partial}{\partial t} H_{n}^{(E)}(x, t)=\left(\mathcal{H}-E_{0}\right) H_{n}^{(E)}(x, t)
$$

с начальным значением $H_{n}^{(E)}(x, 0)=x^{n}$. Из условия коммутирования $[S, K]=0$ получаем, что эволюция по времени оператора $K$ удовлетворяет соотношению

$$
i \hbar \frac{\partial K}{\partial t}=[\mathcal{H}, K]
$$

и имеет вид

$$
K(t)=e^{-i \mathcal{H} t / \hbar} K(0) e^{i \mathcal{H} t / \hbar}=e^{-i \mathcal{H} t / \hbar} x e^{i \mathcal{H} t / \hbar} .
$$

Тогда оператор $K$ порождает бесконечную иерархию многочленов:

$$
K H_{n}(x, t)=K \exp \left[-\frac{i}{\hbar}\left(\mathcal{H}-E_{0}\right) t\right] x^{n}=\exp \left[-\frac{i}{\hbar}\left(\mathcal{H}-E_{0}\right) t\right] x^{n+1}=H_{n+1}(x, t) .
$$

1.1. Нерелятивистское уравнение Шредингера. Из нерелятивистского закона дисперсии $E(p)=p^{2} / 2 m$ вытекает существование оператора Гамильтона

$$
\mathcal{H}=-\frac{\hbar^{2}}{2 m} \frac{\partial^{2}}{\partial x^{2}}
$$

и оператора галилеева буста

$$
K=x+i t \frac{\hbar}{m} \frac{\partial}{\partial x} .
$$

С помощью производящей функции

$$
\exp \left[\frac{i}{\hbar}\left(p x-\frac{p^{2}}{2 m} t\right)\right]=\sum_{n=0}^{\infty}\left(\frac{i}{\hbar}\right)^{n} \frac{p^{n}}{n !} H_{n}^{(\mathrm{S})}(x, t)
$$


получаем многочлены Шредингера

$$
H_{n}^{(\mathrm{S})}(x, t)=\exp \left[\frac{i}{\hbar} t \frac{\hbar^{2}}{2 m} \frac{\partial^{2}}{\partial x^{2}}\right] x^{n} .
$$

Если $H_{n}^{(\mathrm{KF})}(x, t)=e^{t d^{2} / d t^{2}} x^{n}-$ многочлен Кампе де Ферье, то $H_{n}^{(\mathrm{S})}(x, t)=$ $H^{(\mathrm{KF})}(x, i \hbar t / 2 m)$, или, в терминах многочленов Эрмита,

$$
H_{n}^{(\mathrm{S})}(x, t)=\left(-\frac{i \hbar}{2 m} t\right)^{n / 2} H_{n}\left(\frac{x}{\sqrt{-2 i \hbar t / m}}\right) .
$$

1.2. Квазирелятивистское уравнение Шредингера. Из релятивистского закона дисперсии $E(p)=\sqrt{m^{2} c^{4}+c^{2} p^{2}}$ следует гамильтониан

$$
\mathcal{H}=m c^{2} \sqrt{1-\frac{\hbar^{2}}{m^{2} c^{2}} \frac{\partial^{2}}{\partial x^{2}}}
$$

и оператор квазирелятивистского буста

$$
K=x+\frac{i \hbar}{m} t \frac{\partial / \partial x}{\sqrt{1-\left(\hbar^{2} / m^{2} c^{2}\right)\left(\partial^{2} / \partial x^{2}\right)}} .
$$

В нерелятивистском пределе при $c \rightarrow \infty$ это выражение переходит в оператор галилеева буста (9). Производящая функция имеет вид релятивистской плоской волны

$$
\exp \left[\frac{i}{\hbar}\left(p x-\left(\sqrt{m^{2} c^{4}+c^{2} p^{2}}-m c^{2}\right) t\right)\right]=\sum_{n=0}^{\infty}\left(\frac{i}{\hbar}\right)^{n} \frac{p^{n}}{n !} H_{n}^{(\mathrm{SRS})}(x, t)
$$

и задает квазирелятивистские многочлены

$$
H_{n}^{(\mathrm{SRS})}(x, t)=\exp \left[-\frac{i}{\hbar} m c^{2} t\left(\sqrt{1-\frac{\hbar^{2}}{m^{2} c^{2}} \frac{\partial^{2}}{\partial x^{2}}}-1\right)\right] x^{n} .
$$

В нерелятивистском пределе $H_{n}^{(\mathrm{SRS})} \rightarrow H_{n}^{(\mathrm{S})}$. Первые три многочлена в точности совпадают с многочленами Шредингера

$$
H_{1}^{(\mathrm{SRS})}(x, t)=x, \quad H_{2}^{(\mathrm{SRS})}(x, t)=x^{2}+i \frac{\hbar}{m} t, \quad H_{3}^{(\mathrm{SRS})}=x^{3}+i \frac{\hbar}{m} 3 x t,
$$

а начиная с четвертого многочлена

$$
H_{4}^{(\mathrm{SRS})}(x, t)=x^{4}+i \frac{\hbar}{m} 6 x^{2} t-\frac{\hbar^{2}}{m^{2}} 3 t^{2}+i \frac{\hbar^{3}}{m^{3} c^{2}} 3 t
$$

появляются релятивистские поправки порядка $1 / c^{2}$. Для комплекснозначной пространственной переменной $x$, как и в $(2+1)$-мерной теории Черна-Саймонса [4] нули этих многочленов описывают движение точечных вихрей в плоскости. Уравнения движения для $N$ вихрей имеют вид

$$
\dot{x}_{k}=\left.\frac{i}{\hbar} \operatorname{Res}\right|_{x=x_{k}} \mathcal{H}\left[\frac{\hbar}{i}\left(\frac{\partial}{\partial x}+\sum_{l=1}^{N} \frac{1}{x-x_{l}}\right)\right] \cdot 1, \quad k=1,2, \ldots, N .
$$


1.3. Релятивистское уравнение Бюргерса-Шредингера. Применяя преобразование Шредингера $\ln \Psi[5], \Psi=e^{\ln \Psi}$, и тождество

$$
e^{-\ln \Psi} \frac{\partial^{n}}{\partial x^{n}} e^{\ln \Psi}=\left(\frac{\partial}{\partial x}+\frac{\partial \ln \Psi}{\partial x}\right)^{n} \cdot 1,
$$

уравнение Шредингера (1) можно переписать в виде

$$
i \hbar \frac{\partial}{\partial t} \ln \Psi=\mathcal{H}\left[-i \hbar\left(\frac{\partial}{\partial x}+\frac{\partial \ln \Psi}{\partial x}\right)\right] \cdot 1 .
$$

Для комплекснозначной функции $\Psi=e^{i F / \hbar}=e^{R+i S / \hbar}$ введем новую комплекснозначную функцию

$$
V=-i \frac{\hbar}{m} \frac{\partial}{\partial x} \ln \Psi=\frac{1}{m} \frac{\partial}{\partial x} F
$$

имеющую размерность скорости. Тогда $F=S-i \hbar R-$ комплекснозначный потенциал, действительная и мнимая части которого определяют классическую и квантовую скорости, $V=V_{\mathrm{c}}+i V_{\mathrm{q}}=S_{x} / m-i \hbar R_{x} / m$. Тем самым уравнение (20) принимает вид комплекснозначного (квантового) уравнения Гамильтона-Якоби

$$
\frac{\partial F}{\partial t}+\mathcal{H}\left(-i \hbar \frac{\partial}{\partial x}+F_{x}\right) \cdot 1=0 .
$$

В классическом (бездисперсионном) пределе при $\hbar \rightarrow 0$ квантовая скорость $V_{\mathrm{q}}$ обращается в нуль, а комплекснозначный потенциал $F$ переходит в вещественный потенциал скорости $S$, играющий роль главной функции Гамильтона. В этом случае уравнение (21) принимает вид классического уравнения Гамильтона-Якоби $\partial S / \partial t+$ $\mathcal{H}(\partial S / \partial x)=0$. Дифференцируя $(20)$, получаем уравнение для комплексной скорости

$$
i \hbar \frac{\partial V}{\partial t}=-i \frac{\hbar}{m} \frac{\partial}{\partial x}\left[\mathcal{H}\left(-i \hbar \frac{\partial}{\partial x}+m V\right) \cdot 1\right] .
$$

Это уравнение оказывается представлением типа жидкости Маделунга для уравнения Шредингера (1). В классическом пределе оно переходит в уравнение Ньютона $m \partial V_{\mathrm{c}} / \partial t=-\partial\left[\mathcal{H}\left(m V_{\mathrm{c}}\right)\right] / \partial x$ или, если использовать форму записи гидродинамического типа, в уравнение

$$
\frac{\partial V_{\mathrm{c}}}{\partial t}+\mathcal{H}^{\prime}\left(m V_{\mathrm{c}}\right) \frac{\partial V_{\mathrm{c}}}{\partial x}=0
$$

которое представляет собой просто производную от классического уравнения Гамильтона-Якоби. Уравнение (23) имеет общее неявное решение $V_{\mathrm{c}}(x, t)=$ $f\left(x-\mathcal{H}^{\prime}\left(m V_{\mathrm{c}}\right) t\right)$, где $f$ - произвольная функция, в котором развивается сингулярность при достижении критического значения по времени, когда производная $\left(V_{\mathrm{c}}\right)_{x}$ обращается в бесконечность.

1.3.1. Нерелятивистское уравнение Бюргерса-Шредингера. Уравнение Шредингера (1) с нерелятивистским гамильтонианом (8) эквивалентно нелинейному уравнению с комплекснозначной скоростью $V$

$$
i \hbar \frac{\partial V}{\partial t}=-\frac{\hbar^{2}}{2 m} \frac{\partial^{2} V}{\partial x^{2}}-i \hbar V \frac{\partial V}{\partial x},
$$


которое будем называть уравнением Бюргерса-Шредингера. В терминах вещественной и мнимой составляющих это уравнение описывает динамику жидкости Маделунга с плотностью $\rho=e^{R}$ и скоростью $V_{\text {c. }}$ В классическом пределе оно сводится к одному вещественному уравнению на классическую скорость $V_{\text {c }}$, а именно к обыкновенному бездисперсионному уравнению Бюргерса.

1.3.2. Квазирелятивистское уравнение Бюргерса-Шредингера. Если начинать с гамильтониана (13), то процедура “бюргеризации", описанная выше (см. (22)), приводит в итоге к квазирелятивистскому уравнению Бюргерса-Шредингера

$$
\frac{1}{c} \frac{\partial V}{\partial t}+c \frac{\partial}{\partial x}\left[\sqrt{1+\frac{1}{m^{2} c^{2}}\left(-i \hbar \frac{\partial}{\partial x}+m V\right)^{2}} \cdot 1\right]=0
$$

В нерелятивистском пределе это уравнение сводится к уравнению (24), релятивистские поправки к которому в первом порядке имеют вид

$$
\begin{aligned}
& i \hbar \frac{\partial V}{\partial t}=-\frac{\hbar^{2}}{2 m} \frac{\partial^{2} V}{\partial x^{2}}-i \hbar V \frac{\partial V}{\partial x}+\frac{1}{8 m^{3} c^{2}}\left[-\hbar^{4} V_{x x x x}\right]+ \\
& +\frac{1}{8 m^{3} c^{2}}\left[-i m \hbar^{3}\left(10 V_{x} V_{x x}+4 V V_{x x x}\right)+m^{2} \hbar^{2}\left(12 V V_{x}^{2}+6 V^{2} V_{x x}\right)+4 i m^{3} \hbar V^{3} V_{x}\right] .
\end{aligned}
$$

В классическом (бездисперсионном) пределе уравнение (25) принимает вид уравнения гидродинамического типа

$$
\left(V_{\mathrm{c}}\right)_{t}+\frac{V_{\mathrm{c}}}{\sqrt{1+V_{\mathrm{c}}^{2} / c^{2}}}\left(V_{\mathrm{c}}\right)_{x}=0 .
$$

В нерелятивистском пределе это уравнение сводится к бездисперсионному уравнению Бюргерса, а первые релятивистские поправки дают

$$
\left(V_{\mathrm{c}}\right)_{t}+V_{\mathrm{c}}\left(V_{\mathrm{c}}\right)_{x}-\frac{1}{2 c^{2}} V_{\mathrm{c}}^{3}\left(V_{\mathrm{c}}\right)_{x}=0 .
$$

Общее неявное решение уравнения (27) имеет вид

$$
V_{\mathrm{c}}(x, t)=f\left(x-\frac{V_{\mathrm{c}} t}{\sqrt{1+V_{\mathrm{c}}^{2} / c^{2}}}\right),
$$

и в течение конечного времени в нем также развивается сингулярность.

\section{4. Преобразование Беклунда для уравнения Бюргерса-Шредингера.} Применяя преобразование буста (см. определение в тексте после уравнения (1)), можно получить из одного решения $\Psi_{1}$ уравнения Шредингера (1) другое решение этого же уравнения в виде

$$
\Psi_{2}=K \Psi_{1}=\left[x-t \mathcal{H}^{\prime}\left(-i \hbar \frac{\partial}{\partial x}\right)\right] \Psi_{1}
$$

Применяя тождество

$$
\Psi^{-1} G\left(-i \hbar \frac{\partial}{\partial x}\right) \Psi=G\left(-i \hbar \frac{\partial}{\partial x}+m V\right) \cdot 1,
$$


справедливое для комплекснозначных скоростей $V_{a}=-(i \hbar / m) \ln \Psi_{a}, a=1,2$, для уравнения (22) получаем преобразование Беклунда

$$
V_{2}=V_{1}-i \frac{\hbar}{m} \frac{\partial}{\partial x} \ln \left[x-t H^{\prime}\left(-i \hbar \frac{\partial}{\partial x}+m V_{1}\right) \cdot 1\right]
$$

В нерелятивистской квантовой механике в случае гамильтониана (8) это преобразование становится комплекснозначным преобразованием Беклунда для уравнения Бюргерса-Шредингера (24)

$$
V_{2}=V_{1}-i \frac{\hbar}{m} \frac{1-\left(V_{1}\right)_{x} t}{x-V_{1} t}
$$

В квазирелятивистской квантовой механике с гамильтонианом (13) для уравнения (25) получается преобразование Беклунда вида

$$
V_{2}=V_{1}-i \frac{\hbar}{m} \frac{\partial}{\partial x} \ln \left(x-\frac{1}{\sqrt{1+1 /\left(m^{2} c^{2}\right)\left(-i \hbar \partial / \partial x+m V_{1}\right)^{2}}} V_{1} t\right)
$$

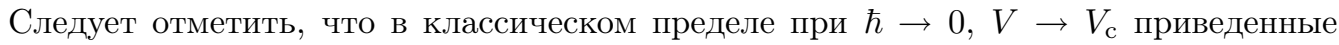
выше преобразования Беклунда сводятся к тривиальному тождеству $V_{\mathrm{c} 1}=V_{\mathrm{c} 2}$.

\section{2. ИНТЕГРИРУЕМАЯ ОБШАЯ ИЕРАРХИЯ НУШ}

В предыдущих разделах было исследовано так называемое $C$-интегрируемое релятивистское уравнение Бюргерса-Шредингера. Теперь построим релятивистское обобщение иерархии НУШ с использованием иерархии Абловица-Каупа-НьюэлаСегура (АКНС) для НУШ.

2.1. Иерархия НУШ. Рассмотрим линейную задачу Захарова-Шабата

$$
\frac{\partial}{\partial x}\left(\begin{array}{l}
v_{1} \\
v_{2}
\end{array}\right)=\left(\begin{array}{cc}
-i p / 2 & -\kappa^{2} \bar{\psi} \\
\psi & i p / 2
\end{array}\right)\left(\begin{array}{l}
v_{1} \\
v_{2}
\end{array}\right)=J_{1}\left(\begin{array}{l}
v_{1} \\
v_{2}
\end{array}\right)
$$

для пространственной эволюции и обобщенную задачу АКНС [6]

$$
\frac{\partial}{\partial t}\left(\begin{array}{l}
v_{1} \\
v_{2}
\end{array}\right)=\left(\begin{array}{cc}
-i A & -\kappa^{2} \bar{C} \\
C & i A
\end{array}\right)\left(\begin{array}{l}
v_{1} \\
v_{2}
\end{array}\right)=J_{0}\left(\begin{array}{l}
v_{1} \\
v_{2}
\end{array}\right)
$$

для временно́й эволюции, где для вещественной функции $A(x, t, p)$ и комплекснозначной функции $C(x, t, p)$, задаваемых условием нулевой кривизны, подстановка $A_{N}=\sum_{n=0}^{N} A^{(n)}(-p / 2)^{n}, C_{N}=\sum_{n=0}^{N} C^{(n)}(-p / 2)^{n}$ приводит к уравнению эволюции

$$
\partial_{t_{N}} \psi=\partial_{x} C^{(0)}+2 i A^{(0)} \psi
$$

с условиями $C^{(N)}=0, A^{(N)}=a_{N}=$ const. Зафиксируем эту константу таким образом, чтобы $a_{N}=(-2)^{N-1}$. Тогда получим рекуррентные соотношения $C^{(n)}=\frac{1}{2 i} \partial_{x} C^{(n+1)}+A^{(n+1)} \psi, \quad \partial_{x} A^{(n)}=i \kappa^{2}\left(\bar{C}^{(n)} \psi-C^{(n)} \bar{\psi}\right), \quad n=0,1,2, \ldots, N-1$. 
Интегрируя последнее уравнение, имеем

$$
A^{(n)}=-i \kappa^{2} \int^{x}\left(\bar{\psi} C^{(n)}-\psi \bar{C}^{(n)}\right)
$$

Подставив (37) в рекуррентное соотношение, получаем

$$
\left(\begin{array}{l}
C^{(n)} \\
\bar{C}^{(n)}
\end{array}\right)=-\frac{1}{2} \mathcal{R}\left(\begin{array}{l}
C^{(n+1)} \\
\bar{C}^{(n+1)}
\end{array}\right)
$$

где $\mathcal{R}$ - матричнозначный интегродифференциальный оператор, который есть не что иное, как рекуррентный оператор иерархии НУШ [6]

$$
\mathcal{R}=i \sigma_{3}\left(\begin{array}{cc}
\partial_{x}+2 \kappa^{2} \psi \int^{x} \bar{\psi} & -2 \kappa^{2} \psi \int^{x} \psi \\
-2 \kappa^{2} \bar{\psi} \int^{x} \bar{\psi} & \partial_{x}+2 \kappa^{2} \bar{\psi} \int^{x} \psi
\end{array}\right),
$$

а $\sigma_{3}$ - третья матрица Паули. Тогда имеем

$$
i \sigma_{3}\left(\begin{array}{c}
\psi \\
\bar{\psi}
\end{array}\right)_{t_{N}}=\mathcal{R}^{N}\left(\begin{array}{c}
\psi \\
\bar{\psi}
\end{array}\right)
$$

где $t_{N}, N=1,2,3, \ldots,-$ бесконечная иерархия времен. В линейном приближении при $\kappa=0$ рекуррентный оператор представляет собой оператор импульса $\mathcal{R}_{0}=$ $i \sigma_{3} \partial / \partial x$, а иерархия НУШ (40) принимает вид иерархии линейного уравнения Шредингера

$$
i \psi_{t_{n}}=i^{n} \partial_{x}^{n} \psi
$$

Представление Маделунга для этой иерархии, задаваемое комплексным преобразованием Коула-Хопфа, обеспечивается комплекснозначной иерархией Бюргерса [4].

Каждое уравнение иерархии (40) интегрируемо. Линейная задача для $N$-го уравнения описывается задачей Захарова-Шабата (35) для пространственной части и задачей

$$
\frac{\partial}{\partial t_{N}}\left(\begin{array}{l}
v_{1} \\
v_{2}
\end{array}\right)=\left(\begin{array}{cc}
-i A_{N} & -\kappa^{2} \bar{C}_{N} \\
C_{N} & i A_{N}
\end{array}\right)\left(\begin{array}{l}
v_{1} \\
v_{2}
\end{array}\right)=J_{0_{N}}\left(\begin{array}{l}
v_{1} \\
v_{2}
\end{array}\right)
$$

для временно́й составляющей. Коэффициентные функции $C_{N}$ могут быть найдены в следующем удобном виде:

$$
\left(\begin{array}{l}
C_{N} \\
\bar{C}_{N}
\end{array}\right)=\sum_{k=1}^{N} p^{N-k} \mathcal{R}^{k-1}\left(\begin{array}{l}
\psi \\
\bar{\psi}
\end{array}\right)=\left(p^{N-1}+p^{N-2} \mathcal{R}+\cdots+\mathcal{R}^{N-1}\right)\left(\begin{array}{l}
\psi \\
\bar{\psi}
\end{array}\right) .
$$

Чтобы записать это выражение в более компактном виде, введем обозначение для оператора в терминах $q$-чисел:

$$
1+q+q^{2}+\cdots+q^{N-1} \equiv[N]_{q}
$$

где $q$ - линейный оператор. Тем самым в терминах оператора $q \equiv \mathcal{R} / p$ получаем следующее конечное разложение Лорана по спектральному параметру $p$ :

$$
1+\frac{\mathcal{R}}{p}+\left(\frac{\mathcal{R}}{p}\right)^{2}+\cdots+\left(\frac{\mathcal{R}}{p}\right)^{N-1} \equiv[N]_{\mathcal{R} / p} .
$$


Тогда имеем

$$
\left(\begin{array}{c}
C_{N} \\
\bar{C}_{N}
\end{array}\right)=p^{N-1}[N]_{\mathcal{R} / p}\left(\begin{array}{c}
\psi \\
\bar{\psi}
\end{array}\right)
$$

Аналогично получаем

$$
A_{N}=-\frac{p^{N}}{2}-i \kappa^{2} p^{N-1}\left(\int^{x} \bar{\psi},-\int^{x} \psi\right)[N]_{\mathcal{R} / p}\left(\begin{array}{c}
\psi \\
\bar{\psi}
\end{array}\right) .
$$

Уравнения (42), (46) и (47) задают временну́ю составляющую линейной задачи (представления Лакса) для $N$-го потока иерархии НУШ (40), выраженного в терминах $q$-анализа.

2.2. Общее уравнение иерархии НУШ. Общее уравнение иерархии НУШ для времени $t$, которое задается формальным рядом $\partial_{t}=\sum_{N=0}^{\infty} E_{N} \partial_{t_{N}}$ с произвольными постоянными $E_{N}$, имеет вид

$$
i \sigma_{3}\left(\begin{array}{c}
\psi \\
\bar{\psi}
\end{array}\right)_{t}=\left(E_{0}+E_{1} \mathcal{R}+\cdots+E_{N} \mathcal{R}^{N}+\cdots\right)\left(\begin{array}{c}
\psi \\
\bar{\psi}
\end{array}\right) .
$$

Интегрируемость этого уравнения связана с задачей Захарова-Шабата (35) и с временно́й эволюцией

$$
J_{0}=\sum_{N=0}^{\infty} E_{N} J_{0_{N}}=\left(\begin{array}{cc}
-i A & -\kappa^{2} \bar{C} \\
C & i A
\end{array}\right)
$$

где

$$
\left(\begin{array}{l}
C \\
\bar{C}
\end{array}\right)=\sum_{N=0}^{\infty} E_{N}\left(\begin{array}{l}
C_{N} \\
\bar{C}_{N}
\end{array}\right)=\sum_{N=1}^{\infty} E_{N} p^{N-1}[N]_{\mathcal{R} / p}\left(\begin{array}{c}
\psi \\
\bar{\psi}
\end{array}\right) ;
$$

здесь было использовано, что для $N=0$ величина $C_{0}=0$. В результате получаем

$$
A=\sum_{N=0}^{\infty} E_{N} A_{N}=-\frac{1}{2} \sum_{N=0}^{\infty} E_{N} p^{N}-i \kappa^{2}\left(\int^{x} \bar{\psi},-\int^{x} \psi\right)\left(\frac{C}{C}\right) .
$$

Уравнение (48) задает интегрируемое нелинейное расширение линейного уравнения Шредингера (1) с обобщенным аналитическим законом дисперсии. Рассмотрим систему классических частиц с соотношением энергии-импульса $E(p)=E_{0}+E_{1} p+$ $E_{2} p^{2}+\cdots$. Тогда соответствующее волновое уравнение Шредингера, зависящее от времени, принимает вид (1), где гамильтонов оператор получается стандартной подстановкой $p \rightarrow-i \hbar \partial / \partial x$ для импульса в законе дисперсии. Уравнение (1) вместе со своим комплексно-сопряженным уравнением запишем в виде

$$
i \hbar \sigma_{3} \frac{\partial}{\partial t}\left(\begin{array}{l}
\psi \\
\bar{\psi}
\end{array}\right)=H\left(-i \hbar \sigma_{3} \frac{\partial}{\partial x}\right)\left(\begin{array}{l}
\psi \\
\bar{\psi}
\end{array}\right) .
$$

Оператор импульса в этом выражении - это оператор рекурсии в линейном приближении: $\mathcal{R}_{0}=i \sigma_{3} \partial / \partial x$. Таким образом, уравнение $(52)$ оказывается линейным 
уравнением Шредингера с произвольным аналитическим законом дисперсии. Нелинейное интегрируемое расширение уравнения (52) проявляется в виде (48), что соответствует подстановке $\mathcal{R}_{0} \rightarrow \mathcal{R}(\hbar=1)$, так что

$$
i \sigma_{3}\left(\begin{array}{l}
\psi \\
\bar{\psi}
\end{array}\right)_{t}=H(\mathcal{R})\left(\begin{array}{l}
\psi \\
\bar{\psi}
\end{array}\right)
$$

С этой точки зрения стандартная подстановка для классического импульса $p \rightarrow$ $-i \hbar \partial / \partial x$ или, эквивалентно, $p \rightarrow-i \hbar \sigma_{3} \partial / \partial x=\mathcal{R}_{0}$ для уравнения в спинорной форме записи задает квантование в виде линейного уравнения Шредингера. В то же время при подстановке $p \rightarrow \mathcal{R}$ получаем “нелинейное квантование" и уравнение из иерархии НУШ.

Представление Лакса, связанное с уравнением (53), задается формулами (50), (51). По определению $q$-производной

$$
D_{q}^{(\zeta)} f(\zeta)=\frac{f(q \zeta)-f(\zeta)}{(q-1) \zeta}
$$

для оператора $q=\mathcal{R} / p$ получаем соотношение $D_{\mathcal{R} / p}^{(p)} \zeta^{N}=[N]_{\mathcal{R} / p} p^{N-1}$. Тогда уравнение (50) можно переписать в виде

$$
\left(\frac{C}{C}\right)=\sum_{N=1}^{\infty} E_{N} p^{N-1}[N]_{\mathcal{R} / p}\left(\begin{array}{c}
\psi \\
\bar{\psi}
\end{array}\right)=\sum_{N=1}^{\infty} E_{N} D_{\mathcal{R} / p}^{(p)} p^{N}\left(\begin{array}{c}
\psi \\
\bar{\psi}
\end{array}\right)
$$

или, учитывая линейность $q$-производной и конкретный вид аналитической дисперсии,

$$
\left(\frac{C}{C}\right)=D_{\mathcal{R} / p}^{(p)} \sum_{N=0}^{\infty} E_{N} p^{N}\left(\begin{array}{c}
\psi \\
\bar{\psi}
\end{array}\right)=D_{\mathcal{R} / p}^{(p)} E(p)\left(\begin{array}{c}
\psi \\
\bar{\psi}
\end{array}\right) .
$$

В силу приведенного выше определения выражение (55) сводится к простой формуле

$$
\left(\frac{C}{C}\right)=\frac{E(\mathcal{R})-E(p)}{\mathcal{R}-p}\left(\begin{array}{l}
\psi \\
\bar{\psi}
\end{array}\right)
$$

Тогда для функции $A$ получаем

$$
A=-\frac{1}{2} E(p)-i \kappa^{2}\left(\int^{x} \bar{\psi},-\int^{x} \psi\right) \frac{E(\mathcal{R})-E(p)}{\mathcal{R}-p}\left(\begin{array}{l}
\psi \\
\bar{\psi}
\end{array}\right) .
$$

Уравнения (56) и (57) задают представление Лакса для общей интегрируемой модели иерархии НУШ (53) в простом и компактном виде. Стоит отметить, что конкретный вид закона дисперсии $E=E(p)$ определяется рассматриваемой физической задачей. В разделе 3 рассмотрим релятивистскую форму этого закона дисперсии и соответствующее квазирелятивистское НУШ. 


\section{3. КВАЗИРЕЛЯТИВИСТСКОЕ НУШ}

В п. 1.2 рассматривался релятивистский закон дисперсии $E(p)=\sqrt{m^{2} c^{4}+p^{2} c^{2}}$. С его помощью можно построить квазирелятивистское уравнение Шредингера с гамильтонианом (13). Тогда, объединяя два комплексно-сопряженных уравнения, получаем

$$
i \sigma_{3}\left(\begin{array}{c}
\psi \\
\bar{\psi}
\end{array}\right)_{t}=m c^{2} \sqrt{1+\frac{1}{m^{2} c^{2}}\left(i \sigma_{3} \frac{\partial}{\partial x}\right)^{2}}\left(\begin{array}{l}
\psi \\
\bar{\psi}
\end{array}\right) .
$$

Заметим, что если $\psi$ описывает движение релятивистской частицы в положительном направлении времени и с положительной энергией, то волновая функция $\bar{\psi}$ отвечает движению в отрицательном направлении времени и с отрицательной энергией. С этой точки зрения уравнение (58) оказывается полным, так как оно описывает оба случая одновременно.

В соответствии с общей процедурой, изложенной в разделе 2 , можно заменить оператор производной $\mathcal{R}_{0}=i \sigma_{3} \partial / \partial x$, отвечающий линейному импульсу $p$, на полный рекуррентный оператор $\mathcal{R}(39)$, что в итоге приведет к интегрируемому релятивистскому нелинейному уравнению Шредингера

$$
i \sigma_{3}\left(\begin{array}{l}
\psi \\
\bar{\psi}
\end{array}\right)_{t}=m c^{2} \sqrt{1+\frac{1}{m^{2} c^{2}} \mathcal{R}^{2}}\left(\begin{array}{l}
\psi \\
\bar{\psi}
\end{array}\right)
$$

где квадратный корень из операторнозначного выражения имеет смысл формального разложения в ряд, так что

$$
i \sigma_{3}\left(\begin{array}{l}
\psi \\
\bar{\psi}
\end{array}\right)_{t}=m c^{2}\left(1+\frac{1}{2 m^{2} c^{2}} \mathcal{R}^{2}-\frac{1}{8 m^{4} c^{4}} \mathcal{R}^{4}+\frac{1}{16 m^{6} c^{6}} \mathcal{R}^{6}-\cdots\right)\left(\begin{array}{l}
\psi \\
\bar{\psi}
\end{array}\right) .
$$

Для приведенного выше релятивистского закона дисперсии и уравнения (59) получаем следующую линейную задачу:

$$
\begin{aligned}
\frac{\partial}{\partial x}\left(\begin{array}{l}
v_{1} \\
v_{2}
\end{array}\right) & =\left(\begin{array}{cc}
-i p / 2 & -\kappa^{2} \bar{\psi} \\
\psi & i p / 2
\end{array}\right)\left(\begin{array}{l}
v_{1} \\
v_{2}
\end{array}\right), \\
\frac{\partial}{\partial t}\left(\begin{array}{l}
v_{1} \\
v_{2}
\end{array}\right) & =\left(\begin{array}{cc}
-i A & -\kappa^{2} \bar{C} \\
C & i A
\end{array}\right)\left(\begin{array}{l}
v_{1} \\
v_{2}
\end{array}\right),
\end{aligned}
$$

где

$$
\begin{gathered}
\left(\begin{array}{c}
C \\
C
\end{array}\right)=\frac{\sqrt{m^{2} c^{4}+\mathcal{R}^{2} c^{2}}-\sqrt{m^{2} c^{4}+p^{2} c^{2}}}{\mathcal{R}-p}\left(\begin{array}{c}
\psi \\
\bar{\psi}
\end{array}\right), \\
A=-\frac{1}{2} \sqrt{m^{2} c^{4}+p^{2} c^{2}}-i \kappa^{2}\left(\int^{x} \bar{\psi},-\int^{x} \psi\right) \frac{\sqrt{m^{2} c^{4}+\mathcal{R}^{2} c^{2}}-\sqrt{m^{2} c^{4}+p^{2} c^{2}}}{\mathcal{R}-p}\left(\begin{array}{l}
\psi \\
\bar{\psi}
\end{array}\right),
\end{gathered}
$$

а спектральный параметр $p$ имеет смысл классического импульса. Модель (59) представляет собой интегрируемое НУШ с релятивистским законом дисперсии

$$
i \psi_{t}=m c^{2} \sqrt{1-\frac{1}{m^{2} c^{2}} \frac{\partial^{2}}{\partial x^{2}}} \psi+F(\psi),
$$


где нелинейная часть, разложенная в ряд по $1 / c^{2}$, задается как

$$
\begin{aligned}
F(\psi)= & \frac{1}{2 m}\left[-2 \kappa^{2}|\psi|^{2} \psi\right]- \\
& -\frac{1}{8 m^{3} c^{2}}\left[2 \kappa^{2}\left(2\left|\psi_{x}\right|^{2} \psi+4|\psi|^{2} \psi_{x x}+\bar{\psi}_{x x} \psi^{2}+3 \bar{\psi} \psi_{x}^{2}\right)+6 \kappa^{4}|\psi|^{4} \psi\right]+O\left(\frac{1}{c^{4}}\right) .
\end{aligned}
$$

Если разложить в ряд по $1 / c^{2}$ также и дисперсионный член, то в каждом порядке по $1 / c^{2}$ возникнет интегрируемая система. Тем самым наша процедура приводит к интегрируемым релятивистским поправкам к НУШ во всех порядках теории возмущений. Ни одна из известных интегрируемых релятивистских моделей типа модели синус-Гордон или уравнения Лиувилля не обладает таким свойством. Наконец, заметим, что нелинейные релятивистские уравнения, построенные в настоящей работе, отличаются от уравнений, полученных в работах [1]-[3] и в статьях, цитируемых в этих работах. Наши модели могут найти применение при исследовании релятивистских поправок к солитонам, к конденсатам Бозе-Эйнштейна или к другим системам конденсированных сред, описываемым эффективными уравнениями релятивистской природы.

Благодарности. Настоящая работа частично поддержана Izmir Institute of Technology (Turkey) (грант BAP 200 8IYTE25).

\section{Список литературы}

[1] L. J. Nickisch, L. Durand, B. Durand, Phys. Rev. D, 30:3 (1984), 660-670.

[2] J. Fröhlich, E. Lenzmann, Comm. Pure Appl. Math., 60:11 (2007), 1691-1705.

[3] Y. Cho, T. Ozawa, SIAM J. Math. Anal., 38:4 (2006), 1060-1074.

[4] О. К. Пашаев, З. Н. Гюркан, ТМФ, 152:1 (2007), 163-176.

[5] E. Schrödinger, Ann. Phys., 384:4 (1926), 361-376.

[6] M. Ablowitz, D. Kaup, A. Newell, H. Segur, Stud. Appl. Math., 53:4 (1974), 249-315. 